\title{
Dynamical Behavior of a New Epidemiological Model
}

\author{
Zizi Wang ${ }^{1}$ and Zhiming Guo ${ }^{2}$ \\ ${ }^{1}$ School of Mathematics and Information Sciences, Guangzhou University, Guangzhou 510006, China \\ ${ }^{2}$ Key Laboratory of Mathematics and Interdisciplinary Science of Guangdong, Higher Education Institutes, \\ Guangzhou University, Guangzhou 510006, China
}

Correspondence should be addressed to Zhiming Guo; gzm100@sina.com

Received 29 October 2013; Revised 27 December 2013; Accepted 15 January 2014; Published 2 March 2014

Academic Editor: P. G. L. Leach

Copyright (c) 2014 Z. Wang and Z. Guo. This is an open access article distributed under the Creative Commons Attribution License, which permits unrestricted use, distribution, and reproduction in any medium, provided the original work is properly cited.

A new epidemiological model is introduced with nonlinear incidence, in which the infected disease may lose infectiousness and then evolves to a chronic noninfectious disease when the infected disease has not been cured for a certain time $\tau$. The existence, uniqueness, and stability of the disease-free equilibrium and endemic equilibrium are discussed. The basic reproductive number $R_{0}$ is given. The model is studied in two cases: with and without time delay. For the model without time delay, the disease-free equilibrium is globally asymptotically stable provided that $R_{0} \leq 1$; if $R_{0}>1$, then there exists a unique endemic equilibrium, and it is globally asymptotically stable. For the model with time delay, a sufficient condition is given to ensure that the disease-free equilibrium is locally asymptotically stable. Hopf bifurcation in endemic equilibrium with respect to the time $\tau$ is also addressed.

\section{Introduction}

As we know, in the SIR model, the population is divided into three classes, $S, I$, and $R$, where $S$ denotes the number of susceptible individuals, $I$ the number of infective individuals, and $R$ the number of removed individuals, respectively. With respect to the SIR models, many studies have proposed several reasons for the nonlinearity of incidence rate at which susceptible individual becomes infective. In 1978, Capasso and Serio [1] found a saturated incidence rate $h(I)=k I /(1+$ $\alpha I)$, where $h(I)$ tends to a saturation level when $I$ gets large. It is more reasonable than the bilinear incidence rate, because it reflects the behavioral change and crowding effect of the infective individuals and prevents the unboundedness of the contact rate by choosing suitable parameters. In 2007, Xiao and Ruan studied the global SIR model with nonmonotone incidence rate $h(I)=k I /\left(1+\alpha I^{2}\right)$ in [2]. Recently, Yang and Xiao [3] extended this nonlinear incidence rate to $h(I)=$ $k I /\left(1+\alpha I^{h}\right), h \geq 1$, by using standard method.

However, the time period of immunity for many infectious diseases is short, or even they have no immunity. Furthermore, these diseases often lead to some other more dangerous diseases. For examples, Chagas disease, hepatitis $\mathrm{C}$, gonorrhea, and other sexually transmitted diseases may advance through several infective stages and have different ability to transmit these infections in different stages of infection. Their infectivity usually depends on the parasite or viral loads in infected individuals or vectors [4]. For instance, in the case of Chagas disease [5], the acute stage follows the invasion of the blood stream by the protozoan $T$. cruzi. This stage lasts from one to two months and infected individuals may or may not show symptoms of the disease. After the acute phase, the infected individuals enter the chronic stage and stay there for variable duration that lasts from 10 to 20 years. At its end, the disease may follow different paths: some individuals may develop mega syndromes and others may present myocarditis. Myocarditis is the terminal form which causes the highest mortality in the group of individuals between 20 and 50 years of age. In fact, there are some research achievements about this phenomenon [610]. For example, Cai et al. [11] investigated a stage-structured epidemic model with a nonlinear incidence and a factor $S^{p}$.

Motivated by the above discussions, we introduce a new epidemiological model in this paper. In this model, the population is divided into three classes, $S, I$, and $D$, where $S(t)$ is the number of susceptible individuals, $I(t)$ the number of infective individuals, and $D(t)$ the number of individuals who are pathologically changed from the infective individuals not being cured for a certain time $\tau$ at time $t$, respectively. 
Our model to be considered takes the following form:

$$
\begin{gathered}
S^{\prime}(t)=b-d S(t)-\frac{k I(t) S(t)}{1+\alpha I(t)}+u_{1} I(t)+u_{2} D(t), \\
I^{\prime}(t)=\frac{k I(t) S(t)}{1+\alpha I(t)}-m e^{-d \tau} I(t-\tau)-\left(u_{1}+d\right) I(t), \\
D^{\prime}(t)=m e^{-d \tau} I(t-\tau)-\left(d+u_{2}\right) D(t),
\end{gathered}
$$

where $b$ is the recruitment of the population, $d$ is the natural death rate of the population, $k$ is the proportional constant, $u_{1}$ is the natural recovery rate of the infective individuals $I$, $u_{2}$ is the natural recovery rate of the disease $D$ (by $D$, we also denote the disease induced by infective disease $I$ ), and $\tau$ is the average time period from infectious disease $I$ evolving to noninfectious disease $D . m$ is the rate by which infective individuals $I$ change into noninfectious individuals $D$, and $\alpha$ is the parameter that measures the psychological or inhibitory effect. $e^{-d \tau}$ is the probability that an individual during the incubation period time $\tau$ survived to develop the disease $D$ and did not emigrate [12]. For simplicity, we set $c=m e^{-d \tau}$. We only consider the system in the first quadrant due to the epidemiological meaning.

It is easy to see that system (1) always has a diseasefree equilibrium $E_{0}=(b / d, 0,0)$. To find the other positive equilibria, set

$$
\begin{gathered}
b-d S-\frac{k I S}{1+\alpha I}+u_{1} I+u_{2} D=0, \\
\frac{k I S}{1+\alpha I}-c I-\left(u_{1}+d\right) I=0, \\
c I-\left(d+u_{2}\right) D=0 .
\end{gathered}
$$

By direct computation, we have

$$
\begin{aligned}
& {\left[\left(u_{2}+d\right) \alpha d\left(u_{1}+c+d\right)+k d\left(u_{2}+c+d\right)\right] I} \\
& +\left(u_{2}+d\right)\left[d\left(u_{1}+c+d\right)-k b\right]=0
\end{aligned}
$$

Then, system (1) has exactly a positive equilibrium $\left(S_{*}, I_{*}, D_{*}\right)$ if and only if $d\left(u_{1}+c+d\right)-k b<0$ with

$$
\begin{gathered}
S_{*}=\frac{\left(c+d+u_{1}\right)\left(1+\alpha I_{*}\right)}{k}, \\
I_{*}=\frac{\left(u_{2}+d\right)\left[k b-d\left(u_{1}+c+d\right)\right]}{\left(u_{2}+d\right) \alpha d\left(u_{1}+c+d\right)+k d\left(u_{2}+c+d\right)}, \\
D_{*}=\frac{c I_{*}}{d+u_{2}} .
\end{gathered}
$$

For system (1) with $\tau=0$, define the basic reproduction number [13] as follows:

$$
R_{0}=\frac{k b}{d\left(u_{1}+c+d\right)} .
$$

By (2) and (3), it is easy to see that
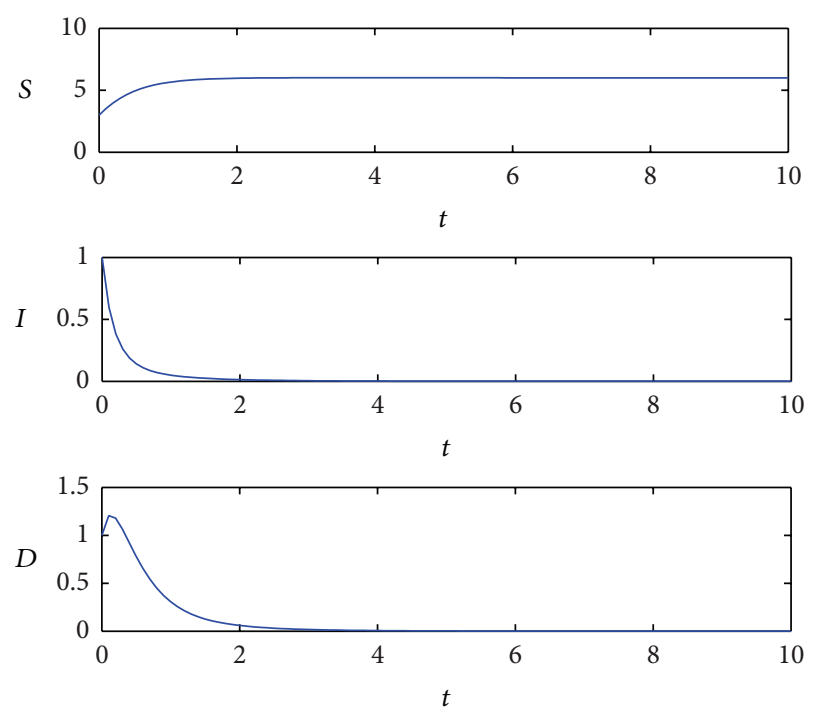

FIGURE 1: When $\tau=0, b=6, \alpha=d=k=1, c=4$, and $u_{1}=u_{2}=2$, then $R_{0}=0.8571<1$. Taking the initial value $(3,1,1)$, then the solution $(S(t), I(t), D(t))$ approaches to the disease-free equilibrium $E_{0}=(6,0,0)$ as $t \rightarrow \infty$.

(i) if $R_{0} \leq 1$, then system (1) has no positive equilibrium;

(ii) if $R_{0}>1$, then system (1) has a unique positive equilibrium $E_{*}=\left(S_{*}, I_{*}, D_{*}\right)$, which is called the endemic equilibrium;

(iii) the equilibrium $E_{0}$ and the equillibrium $E_{*}$ are on the hyperplane $S+I+D=b / d$.

The rest of this paper is organized as follows. In Section 2, we will study the stability of disease-free equilibrium and endemic equilibrium, respectively, of the system without time delay. In Section 3, the system with time delay is considered. The stability conditions for disease-free equilibrium and endemic equilibrium are investigated see Figures 1, 2, 3, and 4. Hopf bifurcation of the endemic equilibrium at a critical time $\tau$ is studied. Finally, some conclusions and discussions will be given in the last section.

\section{The System without Time Delay}

If $\tau=0$, system (1) becomes the following form:

$$
\begin{gathered}
S^{\prime}(t)=b-d S(t)-\frac{k I(t) S(t)}{1+\alpha I(t)}+u_{1} I(t)+u_{2} D(t), \\
I^{\prime}(t)=\frac{k I(t) S(t)}{1+\alpha I(t)}-c I(t)-\left(u_{1}+d\right) I(t), \\
D^{\prime}(t)=c I(t)-\left(d+u_{2}\right) D(t) .
\end{gathered}
$$

In this case, we neglect the time from infectious disease $I$ evolving to noninfectious disease $D$, and by $c$ we denote the rate of disease $I$ changed into disease $D$. 

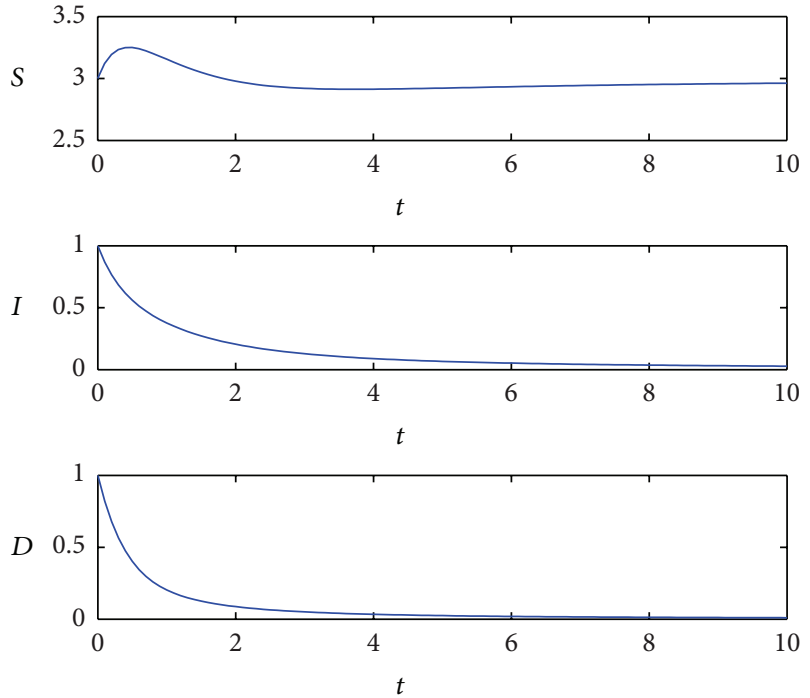

Figure 2: When $\tau=0, b=3, \alpha=d=k=c=u_{1}=1$, $u_{2}=2$, and the initial value is $(3,1,1)$, then $R_{0}=1$, and the solution $(S(t), I(t), D(t))$ approaches to the disease-free equilibrium $E_{0}=(3,0,0)$ as $t \rightarrow \infty$.

2.1. Local Stability of Equilibria. In order to examine local stability of an equilibrium, we should compute the eigenvalues of the linearized operator for system (6) at the equilibrium.

Consider disease-free equilibrium $E_{0}$; the characteristic equation is obtained by the standard method as follows:

$$
(\lambda+d)\left(\lambda+d+u_{2}\right)\left(\lambda-\frac{k b}{d}+u_{1}+d+c\right)=0
$$

It is obvious that $\lambda_{1}=-d<0, \lambda_{2}=-d-u_{2}<0$, and $\lambda_{3}=\left(u_{1}+c+d\right)\left(R_{0}-1\right)$ are the characteristic roots of $(7)$. Thus, we have the following theorem.

Theorem 1. The local stability of disease-free equilibrium $E_{0}$ has the following conclusions as $\tau=0$.

(i) If $R_{0}>1$, then the disease-free equilibrium $E_{0}$ is unstable.

(ii) If $R_{0}<1$, then the disease-free equilibrium $E_{0}$ is locally asymptotically stable.

(iii) If $R_{0}=1$, then the disease-free equilibrium $E_{0}$ is degenerated.

Now, the local stability of the endemic equilibrium $E_{*}=\left(S_{*}, I_{*}, D_{*}\right)$ is considered. As we know, the endemic equilibrium $E_{*}$ exists if and only if $R_{0}>1$. By computation, the characteristic equation of (6) at $E_{*}$ becomes

$$
\lambda^{3}+A \lambda^{2}+B \lambda+C=0
$$
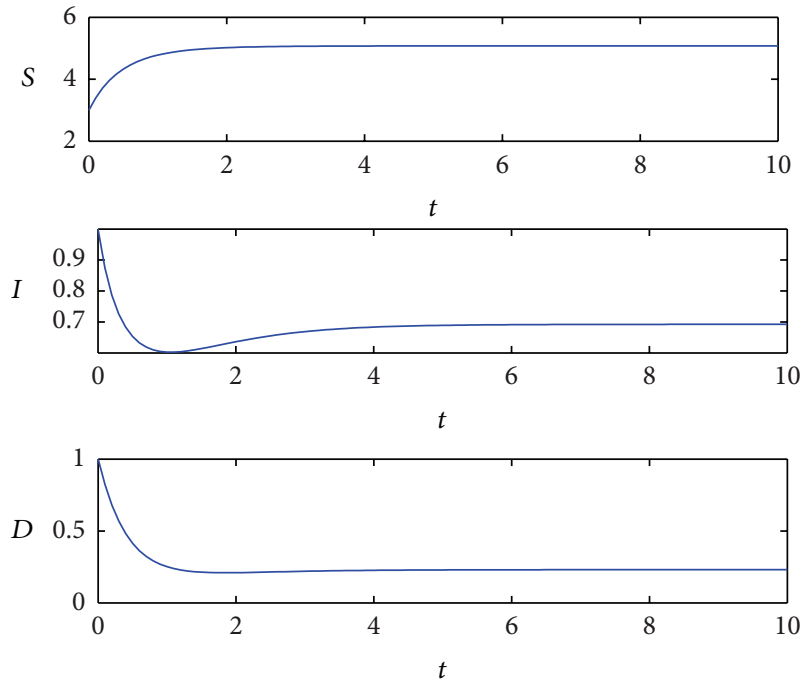

FIgURE 3: Taking the initial value $(3,1,1)$ and $\tau=0, b=6$, $\alpha=d=k=c=u_{1}=1$, and $u_{2}=2$, then $R_{0}=2>1$, and the solution $(S(t), I(t), D(t))$ tends to the endemic equilibrium $E_{*}=(5.0769,0.6923,0.2308)$ as $t \rightarrow \infty$.

where

$$
\begin{gathered}
A=2 d+u_{2}+\frac{k I_{*}}{1+\alpha I_{*}}+\left(u_{1}+c+d\right) \frac{\alpha I_{*}}{1+\alpha I_{*}}>0 \\
B=d^{2}+u_{2} d+\left(2 d+u_{2}\right)\left(u_{1}+c+d\right) \frac{\alpha I_{*}}{1+\alpha I_{*}} \\
+\left(2 d+u_{2}+c\right) \frac{k I_{*}}{1+\alpha I_{*}}>0 \\
C=d\left(u_{2}+d\right)\left(u_{1}+c+d\right) \frac{\alpha I_{*}}{1+\alpha I_{*}} \\
+d\left(u_{2}+d+c\right) \frac{k I_{*}}{1+\alpha I_{*}}>0 .
\end{gathered}
$$

By calculation, we see that $A B>C$. Hence, all roots of (8) have negative real parts by the Routh-Hurwitz criterion. Therefore, we obtain the following result on the locally asymptotic stability of the endemic equilibrium.

Theorem 2. If $R_{0}>1$, then the endemic equilibrium $E_{*}$ is locally asymptotically stable.

2.2. Global Stability of Equilibria. To study the global stability of an equilibrium, we first present two lemmas.

Lemma 3. There exists a positively invariant set $\Lambda$ for system (6), where

$$
\Lambda=\left\{(S, I, D) \in R_{+}^{3}: S \geq 0, I \geq 0, D \geq 0, S+I+D \leq \frac{b}{d}\right\} .
$$



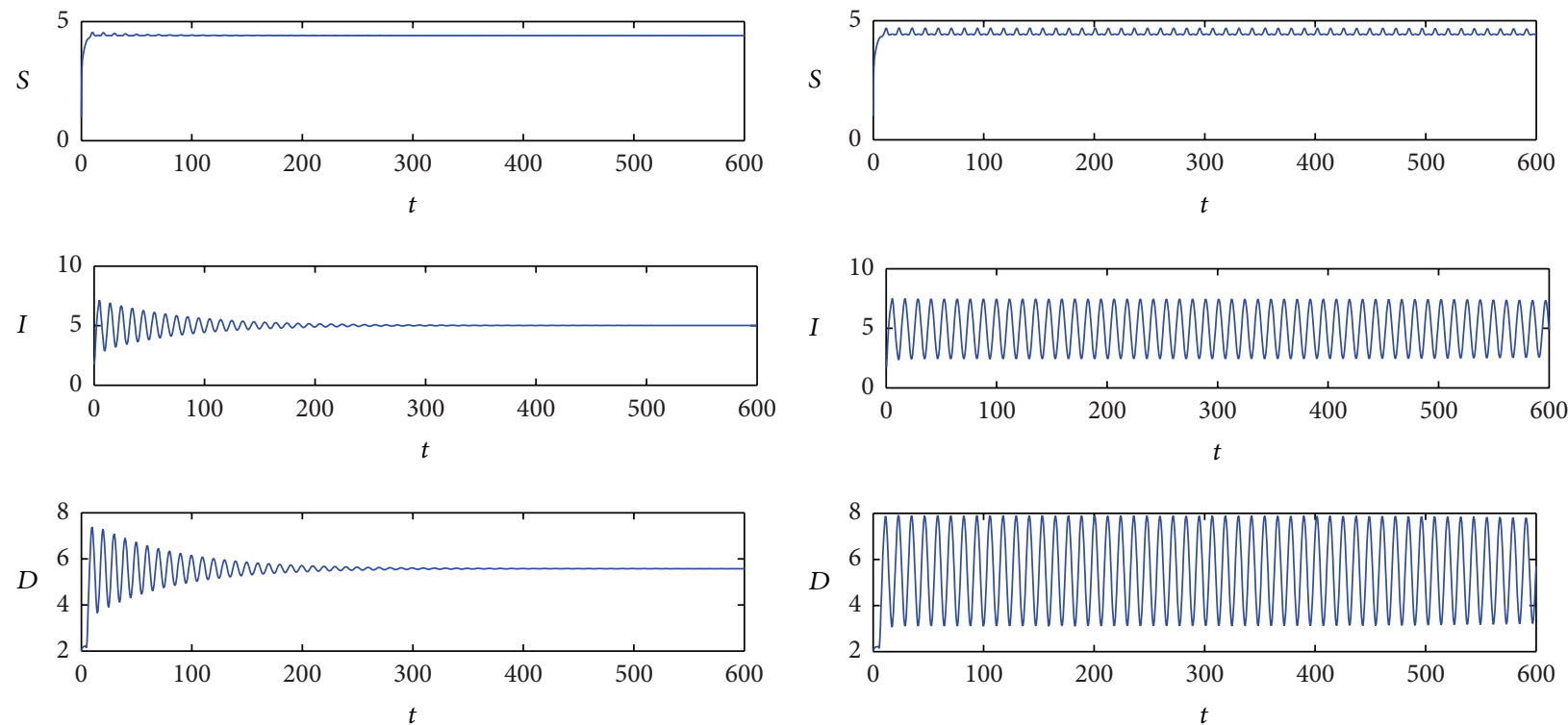

(a) When $\tau=4$, then the solution $(S(t), I(t), D(t))$ converges to the endemic equilibrium $E_{*}=(4.4138,5.0136,5.726)$ as $t \rightarrow \infty$

(b) When $\tau=4.8924$, then a periodic orbit appears in the small neighborhood of the endemic equilibrium $E_{*}=(4.4138,5.0136,5.726)$

Figure 4: Taking $b=6, \alpha=1, d=0.4, k=3, c=1, u_{1}=0.8$, and $u_{2}=0.5$, then $R_{0}=20.4545>1, p_{1}=-6.9925<0, q_{1}=-8.2363<0$, and $r_{1}=-0.4091<0$. And set the initial condition $(S(\theta), I(\theta), D(\theta))=(1,2,2), \theta \in[-\tau, 0]$.

That is, if the initial value $(S(0), I(0), D(0)) \in \Lambda$, then the solutions of (6) are in the set $\Lambda$, for all $t \geq 0$.

Proof. If the initial value $(S(0), I(0), D(0)) \in \Lambda$, then the solution of (6) locally exists and is unique by standard theory on ordinary differential equations [14].

By (6), it is easy to see that on the surface $S(t)=0, d S / d t>$ 0 , if $(0, I(0), D(0)) \in \Lambda$, on the surface $I(t)=0, d I / d t=0$, if $(S(t), 0, D(t)) \in \Lambda$, and on the surface $D(t)=0, d D / d t \geq 0$, if $(S(t), I(t), 0) \in \Lambda$. Hence, no solution of system (6) can exit from the boundary $S=0, I=0, D=0$, if $(S(0), I(0), D(0)) \in$ $\Lambda[15]$. obtain

By adding the first three equations of system (6), we

$$
\frac{d N}{d t}=b-d N(t)
$$

where $N(t)=S(t)+I(t)+D(t)$. Thus,

$$
N(t)=\frac{b}{d}-\left(\frac{b}{d}-N(0)\right) e^{-d t}
$$

If the initial value $(S(0), I(0), D(0)) \in \Lambda$ and $0<N(0) \leq b / d$, then $0<N(t) \leq b / d$. Hence, the solution $(S(t), I(t), D(t))$ of system (6) cannot blow up to infinite for all $t \geq 0$.

In summary, if the initial value $(S(0), I(0), D(0)) \in \Lambda$, then the solution of (6) is in $\Lambda$, for all $t \geq 0$.

It is clear that the limit set of system (6) is on the plane $S+I+D=b / d$. Thus, we have the following lemma.
Lemma 4. The limit set of system (6) in $\Lambda$ is equivalent to that of the following reduced system:

$$
\begin{aligned}
I^{\prime}(t)= & \frac{k I(t)}{1+\alpha I(t)}\left(\frac{b}{d}-I(t)-D(t)\right) \\
& -\left(c+u_{1}+d\right) I(t) \triangleq P(I, D), \\
D^{\prime}(t)= & c I(t)-\left(u_{2}+d\right) D(t) \triangleq Q(I, D),
\end{aligned}
$$

in the triangle region $\Omega=\{(I, D): I \geq 0, D \geq 0, b / d \geq I+D\}$.

One has the following result regarding the nonexistence of periodic orbits in system (13), which implies the nonexistence of periodic orbits of system (6) by Lemmas 3 and 4.

Theorem 5. System (13) does not have nontrivial periodic orbits.

Proof. Consider system (13) for $I \geq 0$ and $D \geq 0$. Take a Dulac function

$$
B(I, D)=\frac{1+\alpha I(t)}{k I(t)}
$$

We have

$$
\begin{aligned}
\frac{\partial(B P)}{\partial I}+\frac{\partial(B Q)}{\partial D}= & -1-\frac{\left(u_{1}+c+d\right) \alpha}{k} \\
& -\frac{1+\alpha I}{k I}\left(u_{2}+d\right)<0 .
\end{aligned}
$$

The conclusion follows. 
Theorem 6. When $\tau=0$, system (1) has the following global dynamics.

(i) If $R_{0} \leq 1$, then system (1) has a unique equilibrium. The disease-free equilibrium $E_{0}$ of system (1) is globally asymptotically stable in the interior of $\Lambda$.

(ii) If $R_{0}>1$, then the system (1) has two equilibria, $E_{0}$ and $E_{*}$. Moreover, the endemic equilibrium $E_{*}$ is globally asymptotically stable in the interior of $\Lambda$.

Proof. (i) It is easy to check the conclusion by Theorem 1(ii), Lemma 3, and Theorem 5 as $R_{0}<1$. Therefore, we only consider the case $R_{0}=1$. For simplicity, we rescale (13) by

$$
x=\frac{k I}{u_{2}+d} I, \quad y=\frac{k D}{u_{2}+d}, \quad s=\left(d+u_{2}\right) t .
$$

Then, we obtain

$$
\begin{gathered}
\frac{d x}{d s}=\frac{x}{1+p x^{2}}(A-x-y)-m x, \\
\frac{d y}{d s}=q x-y,
\end{gathered}
$$

where

$$
\begin{gathered}
p=\frac{\alpha\left(u_{2}+d\right)}{k}, \quad A=\frac{k b}{d\left(d+u_{2}\right)}, \\
m=\frac{c+u_{1}+d}{d+u_{2}}, \quad q=\frac{c}{d+u_{2}} .
\end{gathered}
$$

Clearly, $R_{0}=1$ if and only if $A-m=0$, and system (17) has a unique equilibrium $(0,0)$ in the region $\Omega$. Hence, there exists a small neighborhood $O(\varepsilon)$ of $(0,0)$ such that the dynamics of system (17) is equivalent to that of

$$
\begin{gathered}
\frac{d x}{d s}=-x^{2}-2 x y+O\left(r^{3}\right), \\
\frac{d y}{d s}=q x-y,
\end{gathered}
$$

where $r=\sqrt{x^{2}+y^{2}}$. By Theorem 7.1 in [16] (pp. 114) or Theorem 2.11.1 in [17] (pp. 1500), we know that $(0,0)$ is a saddle-node.

(ii) By Theorem 1(i), Theorem 2, Lemma 3, and Theorem 5, we see that the endemic equilibrium $E_{*}$ is globally asymptotically stable in the interior of $\Lambda$. This completes the proof.

\section{System with Time Delay and Hopf Bifurcation}

3.1. Local Stability of Equilibria. In this section, we consider the system (1) with time delay $\tau>0$. To derive the local stability of equilibrium, we should linearize the system (1) as the form

$$
x^{\prime}(t)=A x(t)+B x(t-\tau)
$$

where $A$ and $B$ are real matrixes. The characteristic equation $[18]$ is

$$
\operatorname{det}\left[\lambda I-A-e^{-\lambda \tau} B\right]=0 .
$$

By this method, we linearize the system (1) at the diseasefree equilibrium $E_{0}$ and obtain the following characteristic equation:

$$
(\lambda+d)\left(\lambda+d+u_{2}\right)\left(\lambda-\frac{k b}{d}+u_{1}+d+c e^{-\lambda \tau}\right)=0 .
$$

It is easy to see that $\lambda_{1}=-d<0$ and $\lambda_{2}=-d-u_{2}<0$ are two characteristic roots of (22). Hence, we only need to discuss the roots of the following equation:

$$
h(\lambda, \tau) \triangleq\left(\lambda-\frac{k b}{d}+u_{1}+d+c e^{-\lambda \tau}\right)=0 .
$$

By discussing (22), we have the following result on the local asymptotic stability of the disease-free equilibrium.

Theorem 7. When $\tau>0$, the disease-free equilibrium $E_{0}$ of system (1) has the following conclusion.

(i) If $R_{0}<R_{1}=(c d+k b) /\left(u_{1} d+d^{2}\right)<1$, then the disease-free equilibrium $E_{0}$ of system (1) is locally asymptotically stable.

(ii) If $R_{0}>1$, then the disease-free equilibrium $E_{0}$ of system (1) is unstable.

(iii) If $R_{0}=1$, then the disease-free equilibrium $E_{0}$ of system (1) is degenerated.

Proof. (i) By implicit function theorem for complex variables, we know that the root of (23) is continuous on the parameter $\tau$.

If $R_{0}<1$, then 0 is not a root of (23), for all $\tau>0$. Note that all complex roots of (23) must come in conjugate pairs and all roots of (23) are negative for $\tau=0$. Thus, all roots of (23) have negative real parts for small $\tau$; that is, $0<\tau \ll 1$. Assume that there exists a positive number $\tau=\tau_{0}$ such that (23) has a pair of purely imaginary roots $\lambda= \pm \omega i$, where $\omega$ is a positive number. Then,

$$
\begin{gathered}
c \cos \omega \tau_{0}=\frac{k b}{d}-u_{1}-d, \\
c \sin \omega \tau_{0}=\omega_{0} .
\end{gathered}
$$

Adding up the square of both equations in (24), we obtain

$$
\omega^{2}=\left(1-R_{0}\right)\left(R_{1}-1\right)\left(u_{1}+c+d\right)\left(d+u_{1}\right) .
$$

When $R_{0}<R_{1}<1$, then $\omega^{2}<0$. It is a contradiction with $\omega^{2}>0$ which leads to the nonexistence of $\tau_{0}$.

(ii) We set $h(\lambda, \tau)=\left(\lambda-(k b / d)+u_{1}+d+c e^{-\lambda r}\right)=0$. When $\lambda=0$ and $R_{0}>1$, then $h(0, \tau)=\left(d+u_{1}+c\right)\left(1-R_{0}\right)<0$ and $\lim _{\lambda \rightarrow+\infty} h(\lambda, \tau) \rightarrow+\infty$. Therefore, (23) must have a positive real root for all $\tau>0$.

(iii) If $R_{0}=1$, it is easy to know that $\lambda=0$ is a root of (23), for all $\tau>0$, which leads to conclusion (iii). This completes the proof of the theorem. 
Now, the local stability of the endemic equilibrium $E_{*}=\left(S_{*}, I_{*}, D_{*}\right)$ is considered. As we know, the endemic equilibrium $E_{*}$ exists if and only if $R_{0}>1$. By computation, the associated transcendental characteristic equation of (1) at $E_{*}$ becomes

$$
\lambda^{3}+A \lambda^{2}+B \lambda+C+\left(A_{1} \lambda^{2}+B_{1} \lambda+C_{1}\right) e^{-\lambda \tau}=0,
$$

where

$$
\begin{gathered}
A=2 d+u_{2}-c+\frac{k I_{*}}{1+\alpha I_{*}}+\left(u_{1}+c+d\right) \frac{\alpha I_{*}}{1+\alpha I_{*}}, \\
A_{1}=c, \\
B=d^{2}-2 c d+u_{2} d-c u_{2} \\
+\left(2 d+u_{2}\right)\left(u_{1}+c+d\right) \frac{\alpha I_{*}}{1+\alpha I_{*}}+\left(2 d+u_{2}\right) \frac{k I_{*}}{1+\alpha I_{*}}, \\
B_{1}=2 c d+u_{2} c+c \frac{k I_{*}}{1+\alpha I_{*}}, \\
C=d\left(d+u_{2}\right)\left(-c+\left(u_{1}+c+d\right) \frac{\alpha I_{*}}{1+\alpha I_{*}}+\frac{k I_{*}}{1+\alpha I_{*}}\right), \\
C_{1}=\left(u_{2}+d\right) d c+c d \frac{k I_{*}}{1+\alpha I_{*}} .
\end{gathered}
$$

When $\tau=0$, (26) becomes

$$
\lambda^{3}+\left(A+A_{1}\right) \lambda^{2}+\left(B+B_{1}\right) \lambda+C+C_{1}=0,
$$

where

$$
\begin{gathered}
A+A_{1}=2 d+u_{2}+\frac{k I_{*}}{1+\alpha I_{*}}+\left(u_{1}+c+d\right) \frac{\alpha I_{*}}{1+\alpha I_{*}}>0 \\
B+B_{1}=d^{2}+u_{2} d+\left(2 d+u_{2}\right)\left(u_{1}+c+d\right) \frac{\alpha I_{*}}{1+\alpha I_{*}} \\
+\left(2 d+u_{2}+c\right) \frac{k I_{*}}{1+\alpha I_{*}}>0 \\
C+C_{1}=d\left(u_{2}+d\right)\left(u_{1}+c+d\right) \frac{\alpha I_{*}}{1+\alpha I_{*}} \\
+d\left(u_{2}+d+c\right) \frac{k I_{*}}{1+\alpha I_{*}}>0 .
\end{gathered}
$$

Thus, we obtain the following result on the local asymptotic stability of the endemic equilibrium.

Theorem 8. If $R_{0}>1, c \leq u_{2}, 2 c \leq d$,

$$
\frac{2 k c\left(R_{0}-1\right)\left(u_{2}+c+d\right)}{\left[\left(u_{1}+d-c\right)\left(R_{0}-1\right)-2 c\right]\left(u_{2}+d\right)\left(R_{0}-1\right)\left(u_{1}+d+c\right)} \leq \alpha,
$$

and $0<\left(u_{1}+d-c\right)\left(R_{0}-1\right)-2 c$, then the endemic equilibrium $E_{*}$ of system (1) is locally asymptotically stable for $\tau>0$.
Proof. By implicit function theorem for complex variables, we know that the root of (23) is continuous on the parameter $\tau$. If $R_{0}>1$, then 0 is not a root of (26) for all $\tau>0$. Note that all complex roots of (26) must come in conjugate pairs and all roots of (26) have negative real parts as $\tau=0$ by Theorem 2 . Thus, all roots of (26) have negative real parts for very small $\tau, 0<\tau \ll 1$. Suppose that there exists a positive $\tau_{0}$ such that (26) has a pair of purely imaginary roots $\pm \omega, \omega>0$. Then, $\omega>0$ satisfies

$$
\begin{aligned}
& -i\left(\omega^{3}+A \omega^{2}-B \omega\right)+C \\
& \quad+\left(C_{1}-A_{1} \omega^{2}+i B_{1} \omega\right)\left(\cos \omega \tau_{0}-i \sin \omega \tau_{0}\right)=0 .
\end{aligned}
$$

Separating the real and imaginary parts, we have

$$
\begin{aligned}
& \left(C_{1}-A_{1} \omega^{2}\right) \cos \omega \tau_{0}+B_{1} \omega \sin \omega \tau_{0}=A \omega^{2}-C, \\
& B_{1} \omega \cos \omega \tau_{0}-\left(C_{1}-A_{1} \omega^{2}\right) \sin \omega \tau_{0}=\omega^{3}-B \omega,
\end{aligned}
$$

which implies that

$$
\begin{aligned}
\omega^{6} & +\left(A^{2}-2 B-A_{1}^{2}\right) \omega^{4} \\
& +\left(B^{2}+2 A_{1} C_{1}-2 A C-B_{1}^{2}\right) \omega^{2}+C^{2}-C_{1}^{2}=0 .
\end{aligned}
$$

Let $z=\omega^{2}$. Then, (33) becomes

$$
z^{3}+p_{1} z^{2}+q_{1} z+r_{1}=0
$$

where $p_{1}=A^{2}-2 B-A_{1}^{2}, q_{1}=B^{2}+2 A_{1} C_{1}-2 A C-B_{1}^{2}$, and $r_{1}=C^{2}-C_{1}^{2}$. By computation, we have

$$
\begin{aligned}
p_{1}= & 2 d^{2}+2 u_{2} d+u_{2}^{2}-2 c x-2 c y+x^{2}+2 x y+y^{2} \\
\geq & 7 c^{2}+2 u_{2} d+u_{2}^{2}+\left(c^{2}-2 c x+x^{2}\right)+2 x y \\
& +(y-2 c) y \\
= & 7 c^{2}+2 u_{2} d+u_{2}^{2}+(c-x)^{2}+2 x y+(y-2 c) y, \\
q_{1}= & \left(u_{2}^{2}+2 u_{2} d+2 d^{2}\right) y(y-2 c) \\
& +\left(4 d^{2}+4 u_{2} d+2 u_{2}^{2}\right) x y+H(x), \\
r_{1}= & {\left[d\left(u_{2}+d-c\right) x\right.} \\
& \left.+d\left(u_{2}+d\right)(y-2 c),\left(u_{2}+d\right) d(x+y)+c d x\right],
\end{aligned}
$$

where $x=k I_{*} /\left(1+\alpha I_{*}\right), y=\left(u_{1}+c+d\right)\left(\alpha I_{*} /\left(1+\alpha I_{*}\right)\right)$, and

$$
\begin{aligned}
H(x)= & \left(2 d^{2}+2 u_{2} d+u_{2}^{2}-c^{2}\right) x^{2} \\
& -\left(4 c d^{2}+2 u_{2}^{2} c+2 u_{2} c^{2}+4 c d u_{2}+2 c^{2} d\right) x \\
& +u_{2}^{2} d^{2}+d^{4}+2 d^{3} u_{2} .
\end{aligned}
$$

It is easy to check that if $y>2 c$ and $H(x)>0$, then $p_{1}>0$, $q_{1}>0$, and $r_{1}>0$. 
Now, we examine the sign of $H(x)$ as follows:

$$
\begin{aligned}
& H(x)=\left(2 d^{2}+2 u_{2} d+u_{2}^{2}-c^{2}\right) x^{2} \\
& -\left(4 c d^{2}+2 u_{2}^{2} c+2 u_{2} c^{2}+4 c d u_{2}+2 c^{2} d\right) x \\
& +u_{2}^{2} d^{2}+d^{4}+2 d^{3} u_{2} \\
& \geq\left(7 c^{2}+c d+u_{2} d+u_{2}^{2}\right) x^{2} \\
& -\left(4 c d^{2}+2 u_{2}^{2} c+2 u_{2} c^{2}+4 c d u_{2}+2 c^{2} d\right) x \\
& +d^{4}+2 u_{2}^{2} c^{2}+\frac{1}{2} u_{2}^{2} d^{2}+2 d^{3} c \\
& =\left(2 c x-d^{2}\right)^{2}+\left(u_{2} x-u_{2} c\right)^{2}+\left(c x-u_{2} c\right)^{2} \\
& +\left(\sqrt{c d} x-\sqrt{c^{3} d}\right)^{2} \\
& +\left(2 c^{2}+u_{2} d\right) x^{2}-4 c d u_{2} x+\frac{1}{2} u_{2}^{2} d^{2}+d^{3} c \\
& \geq\left(2 c x-d^{2}\right)^{2}+\left(u_{2} x-u_{2} c\right)^{2}+\left(c x-u_{2} c\right)^{2} \\
& +\left(\sqrt{c d} x-\sqrt{c^{3} d}\right)^{2}+4 c^{2} x^{2}-4 c d u_{2} x \\
& +\frac{1}{2} u_{2}^{2} d^{2}+d^{3} c \\
& \geq\left(2 c x-d^{2}\right)^{2}+\left(u_{2} x-u_{2} c\right)^{2}+\left(c x-u_{2} c\right)^{2} \\
& +\left(\sqrt{c d} x-\sqrt{c^{3} d}\right)^{2}+\left(\sqrt{8} c x-\sqrt{\frac{1}{2}} u_{2} d\right)^{2}+d^{3} c
\end{aligned}
$$

$\geq 0$.

By (2), we know that $I_{*}=\left(\left(u_{1}+c+d\right)\left(u_{2}+d\right)\left(R_{0}-\right.\right.$ $1)) /\left(\alpha\left(u_{2}+d\right)\left(u_{1}+c+d\right)+k\left(u_{2}+c+d\right)\right)$. Now, we set $y>2 c$; then it follows that

$$
\begin{gathered}
\frac{1}{\alpha I_{*}}+1 \leq \frac{u_{1}+c+d}{2 c}, \quad \frac{1}{\alpha I_{*}} \leq \frac{u_{1}+d-c}{2 c} \\
\frac{1}{R_{0}-1}+\frac{k\left(u_{2}+c+d\right)}{\alpha\left(u_{2}+d\right)\left(u_{1}+c+d\right)\left(R_{0}-1\right)} \leq \frac{u_{1}+d-c}{2 c} \\
\frac{k\left(u_{2}+c+d\right)}{\alpha\left(u_{2}+d\right)\left(u_{1}+c+d\right)\left(R_{0}-1\right)} \leq \frac{\left(u_{1}+d-c\right)\left(R_{0}-1\right)-2 c}{2 c\left(R_{0}-1\right)} \\
\frac{2 k c\left(R_{0}-1\right)\left(u_{2}+c+d\right)}{\left[\left(u_{1}+d-c\right)\left(R_{0}-1\right)-2 c\right]\left(u_{2}+d\right)\left(R_{0}-1\right)\left(u_{1}+d+c\right)} \leq \alpha .
\end{gathered}
$$

Now that $p_{1}>0, q_{1}>0$, and $r_{1}>0$ under the conditions of this theorem, (34) has no positive roots, which implies the nonexistence of $\tau_{0}$. Thus, all roots of (26) have negative real parts for $\tau>0$. The proof is finished.
3.2. Hopf Bifurcation. In what follows, we consider the Hopf bifurcation of the disease-free equilibrium $E_{0}$.

Theorem 9. If $\tau>0, R_{0}<1$, and $R_{1}=(c d+k b) /\left(u_{1} d+\right.$ $\left.d^{2}\right)>1$, then there exists a pair of purely imaginary eigenvalues $\pm \omega_{0} i$ as $\tau=\tau_{0}$, and the disease-free equilibrium of system (1) is locally asymptotically stable as $0<\tau<\tau_{0}$. On the other hand, system (1) can undergo a Hopfbifurcation if $\tau>\tau_{0}$, and a periodic orbit appears in the small neighborhood of the diseasefree equilibrium $E_{0}$.

Proof. When $R_{1}>1$, we obtain $\omega_{0}$ from (25). Let

$$
\tau_{0}=\frac{1}{\omega_{0}}\left(\arcsin \frac{\omega_{0}}{c}\right) .
$$

By the implicit function theorem for complex variables, the first conclusion is completely proved.

Suppose that $\lambda(\tau)=\sigma(\tau)+i \omega(\tau)$ is a root of (23) as $\tau>\tau_{0}$. Differentiating (23) with respect to $\tau$, we obtain

$$
\frac{d \lambda}{d \tau}=\frac{c \lambda e^{-\lambda \tau}}{1-c \tau e^{-\lambda \tau}}
$$

Note that $\lambda\left(\tau_{0}\right)= \pm \omega_{0}$. We consider

$$
\begin{aligned}
\operatorname{Re}\left[\frac{d \tau}{d \lambda\left(\tau_{0}\right)}\right] & =\operatorname{Re}\left[\frac{1}{c \lambda e^{-\lambda \tau}}-\frac{\tau}{\lambda}\right]_{\tau=\tau_{0}} \\
& =\operatorname{Re}\left[\frac{1}{c i \omega_{0}\left(\cos \omega_{0} \tau_{0}-i \sin \omega_{0} \tau_{0}\right)}-\frac{\tau_{0}}{i \omega_{0}}\right] \\
& =\operatorname{Re}\left[\frac{\cos \omega_{0} \tau_{0}+i \sin \omega_{0} \tau_{0}}{c i \omega_{0}}\right]=\frac{\sin \omega_{0} \tau_{0}}{c \omega_{0}} .
\end{aligned}
$$

By (24), we have that $\sin \omega_{0} \tau_{0} / c \omega_{0}=1 / c^{2}>0$. Thus,

$$
\operatorname{Re}\left[\frac{d \lambda}{d \tau}\right]_{\tau=\tau_{0}}>0
$$

which leads to $\operatorname{Re}(\lambda(\tau))>0$ as $\tau>\tau_{0}$. The proof is finished.

Now, we consider the Hopf bifurcation of the endemic equilibrium $E_{*}$.

By Theorem 9, we know that the endemic equilibrium $E_{*}$ is locally asymptotically stable for all $\tau>0$ if $c \leq u_{2}, 2 c \leq d$,

$$
\frac{2 k c\left(R_{0}-1\right)\left(u_{2}+c+d\right)}{\left[\left(u_{1}+d-c\right)\left(R_{0}-1\right)-2 c\right]\left(u_{2}+d\right)\left(R_{0}-1\right)\left(u_{1}+d+c\right)} \leq \alpha,
$$

and $0<\left(u_{1}+d-c\right)\left(R_{0}-1\right)-2 c$. Now, we consider the case that $u_{2}+d<c, 0<\left(u_{1}+d-c\right)\left(R_{0}-1\right)-2 c$,

$$
\alpha<\frac{2 k c\left(R_{0}-1\right)\left(u_{2}+c+d\right)}{\left[\left(u_{1}+d-c\right)\left(R_{0}-1\right)-2 c\right]\left(u_{2}+d\right)\left(R_{0}-1\right)\left(u_{1}+d+c\right)},
$$

if $R_{0}>1, \tau>0$. 
Lemma 10. If $R_{0}>1, u_{2}+d<c, 0<\left(u_{1}+d-c\right)\left(R_{0}-1\right)-2 c$, and

$$
\alpha<\frac{2 k c\left(R_{0}-1\right)\left(u_{2}+c+d\right)}{\left[\left(u_{1}+d-c\right)\left(R_{0}-1\right)-2 c\right]\left(u_{2}+d\right)\left(R_{0}-1\right)\left(u_{1}+d+c\right)},
$$

then there exists a pair of purely imaginary eigenvalues $\pm \omega_{0} i$ as $\tau=\tau_{0}$, and the endemic equilibrium $E_{*}$ of system (6) is locally asymptotically stable as $0<\tau<\tau_{0}$.

Proof. By Theorem 9, we know that characteristic equation (26) may have a pair of purely imaginary roots $\pm \omega_{0} i$ for some $\tau>0$ when $R_{0}>1$ and (34) has positive real roots. where

We know that (34) has at least a positive root if $r_{1}<0$,

$$
\begin{aligned}
r_{1}= & {\left[d\left(u_{2}+d-c\right) x+d\left(u_{2}+d\right)(y-2 c)\right] } \\
& \times\left[\left(u_{2}+d\right) d(x+y)+c d x\right] .
\end{aligned}
$$

It is easy to check that $r_{1}<0$ if $u_{2}+d-c<0, y<2 c$. From Theorem 9, if

$$
\alpha<\frac{2 k c\left(R_{0}-1\right)\left(u_{2}+c+d\right)}{\left[\left(u_{1}+d-c\right)\left(R_{0}-1\right)-2 c\right]\left(u_{2}+d\right)\left(R_{0}-1\right)\left(u_{1}+d+c\right)},
$$

then $y<2 c$. Hence, (34) has at least a positive root and at most three different positive real roots under the conditions of this lemma.

Assume that (34) has positive real roots, denoted by $z_{k}$, $k=1,2,3$. Then, (33) has positive roots $\omega_{k}^{2}=z_{k}$. Let

$$
\begin{aligned}
& \tau_{k}^{j} \\
& =\frac{1}{\omega_{k}} \\
& \quad \times\left[\arccos \left(\frac{\left(B_{1}-A A_{1}\right) \omega_{k}^{4}+\left(C_{1} A+A_{1} C-B_{1} B\right) \omega_{k}^{2}-C_{1} C}{A_{1}^{2} \omega_{k}^{4}+\left(B_{1}^{2}-2 A_{1} C_{1}\right) \omega_{k}^{2}+C_{1}^{2}}\right)\right. \\
& \quad+2 j \pi], \quad k=1,2,3 ; j \in Z^{+} .
\end{aligned}
$$

We set

$$
\tau_{*}=\min _{k=1,2,3}\left\{\tau_{k}^{0}\right\}
$$

Then, the characteristic equation (26) of equilibrium $E_{*}$ has a pair of purely imaginary roots $\pm \omega_{0} i$ as $\tau=\tau_{*}$. Note that all roots of (26) have negative real parts as $\tau=0$. Therefore, all roots of (26) have negative real parts as $0<\tau<\tau_{0}$ by continuity of roots on parameter $\tau$. This completes the proof of Lemma 10.

Theorem 11. Assume that the condition of Lemma 10 holds. Then, system (1) can undergo a Hopfbifurcation if $\tau>\tau_{*}$, and a periodic orbit appears in the small neighborhood of the endemic equilibrium $E_{*}$ if either $p_{1} \geq 0$ or $q_{1} \leq 0$.
Proof. We consider the transversal conditions [19] for the complex eigenvalues of the endemic equilibrium $E_{*}$ at $\tau=\tau_{*}$.

Suppose that $\lambda(\tau)=\sigma(\tau)+i \omega(\tau)$ is a root of $(26)$ as $\tau>\tau_{*}$. Differentiating (26) with respect to $\tau$, we obtain [3]

$$
\left[\frac{d \operatorname{Re} \lambda\left(\tau_{*}\right)}{d \tau}\right]^{-1}=\frac{z_{0}}{Z}\left(3 z_{0}^{2}+2 p_{1} z_{0}+q_{1}\right)
$$

where $Z=\left[B_{1}^{2} \omega_{0}^{2}+\left(C_{1}-A_{1} \omega_{0}^{2}\right)^{2}\right] \omega_{0}^{2}>0$.

Since $z^{3}+p_{1} z^{2}+q_{1} z+r_{1}=0$ and $r_{1}<0,3 z_{0}^{2}+2 p_{1} z_{0}+q_{1}>0$ if either $p_{1} \geq 0$ or $q_{1} \leq 0$. Therefore we have

$$
\frac{d \operatorname{Re} \lambda\left(\tau_{*}\right)}{d \tau}>0
$$

if either $p_{1} \geq 0$ or $q_{1} \leq 0$.

By Lemma 10 and Hopf bifurcation theorem in [18], system (6) can undergo a Hopf bifurcation as $\tau>\tau_{*}$, and a periodic orbit appears in the small neighborhood of the endemic $E_{*}$. This completes the proof.

\section{Conclusion}

In this paper, we propose the SID model with time delays. Our purpose is to comprehend the number of disease $D$ which is caused by the infectious disease $I$ in a certain area. For example, hepatitis B causes liver cancer and we want to know the number of liver cancer caused by hepatitis $B$ as $t \rightarrow \infty$. Our analytical results and numerical simulations with different parameter values show that this SID model has some interesting dynamics as follows. When $\tau=0$, the basic reproductive number $R_{0}$ plays a role in the behaviors of this system. If $R_{0} \leq 1$, then the disease $D$ will become extinct as $t \rightarrow \infty$; when $R_{0}>1$, the endemic equilibrium is globally asymptotically stable. Namely, the number of disease $D$ will tend to a certain number and will not die out as $t \rightarrow \infty$. But, for the stability of the time delayed model, we cannot give complete analysis because of the complexity of the system and it is the work in the future. Here, we give some stability analysis in some special case. When $\tau>0, R_{0}<1$, and $R_{1}=(c d+k b) /\left(u_{1} d+d^{2}\right)>1$, the Hopf bifurcation appeared which implies that the disease will appear again even it dies out at this time. We have shown that if $R_{0}>1, u_{2}+d<c$, $0<\left(u_{1}+d-c\right)\left(R_{0}-1\right)-2 c$, and

$$
\alpha<\frac{2 k c\left(R_{0}-1\right)\left(u_{2}+c+d\right)}{\left[\left(u_{1}+d-c\right)\left(R_{0}-1\right)-2 c\right]\left(u_{2}+d\right)\left(R_{0}-1\right)\left(u_{1}+d+c\right)},
$$

then there exists a positive time period $\tau_{0}$ such that the model has a periodic solution as $\tau>\tau_{0}$ which does not appear by bilinear incidence rate [20].

\section{Conflict of Interests}

The authors declare that there is no conflict of interests regarding the publication of this paper. 


\section{Acknowledgments}

The authors are very grateful to the anonymous referees for careful reading and helpful suggestions which led to the improvement of their original paper. This work was partially supported by the National Natural Science Foundation of China, Program for Changjiang Scholars and Innovative Research Team in University (IRT1226), and Research Fund for the Doctoral Program of Higher Education of China (no. 20124410110001).

\section{References}

[1] V. Capasso and G. Serio, "A generalization of the KermackMcKendrick deterministic epidemic model," Mathematical Biosciences, vol. 42, no. 1-2, pp. 43-61, 1978.

[2] D. Xiao and S. Ruan, "Global analysis of an epidemic model with nonmonotone incidence rate," Mathematical Biosciences, vol. 208, no. 2, pp. 419-429, 2007.

[3] Y. Yang and D. Xiao, "Influence of latent period and nonlinear incidence rate on the dynamics of SIRS epidemiological models," Discrete and Continuous Dynamical Systems, vol. 13, no. 1, pp. 195-211, 2010.

[4] R. M. Anderson and R. M. May, Infectious Diseases of Humans, Oxford University Press, London, UK, 1991.

[5] J. X. Velasco-Hernandez, "An epidemiological model for the dynamics of Chagas' disease," Biosystems, vol. 26, no. 2, pp. 127$134,1991$.

[6] H. Guo and M. Y. Li, "Global dynamics of a staged progression model for infectious diseases," Mathematical Biosciences and Engineering, vol. 3, no. 3, pp. 513-525, 2006.

[7] J. M. Hyman, J. Li, and E. A. Stanley, "The differential infectivity and staged progression models for the transmission of HIV," Mathematical Biosciences, vol. 155, no. 2, pp. 77-109, 1999.

[8] J. Li, Y. Zhou, Z. Ma, and J. M. Hyman, "Epidemiological models for mutating pathogens," SIAM Journal on Applied Mathematics, vol. 65, no. 1, pp. 1-23, 2004.

[9] M. Martcheva and C. Castillo-Chavez, "Diseases with chronic stage in a population with varying size," Mathematical Biosciences, vol. 182, no. 1, pp. 1-25, 2003.

[10] S. M. Moghadas and A. B. Gumel, "Global stability of a twostage epidemic model with generalized non-linear incidence," Mathematics and Computers in Simulation, vol. 60, no. 1-2, pp. 107-118, 2002.

[11] L. M. Cai, X. Z. Li, and M. Ghosh, "Global stability of a stagestructured epidemic model with a nonlinear incidence," Applied Mathematics and Computation, vol. 214, no. 1, pp. 73-82, 2009.

[12] Z. Mukandavire, W. Garira, and C. Chiyaka, "Asymptotic properties of an HIV/AIDS model with a time delay," Journal of Mathematical Analysis and Applications, vol. 330, no. 2, pp. 916-933, 2007.

[13] P. van den Driessche and J. Watmough, "Reproduction numbers and sub-threshold endemic equilibria for compartmental models of disease transmission," Mathematical Biosciences, vol. 180, pp. 29-48, 2002.

[14] W. Walter, Ordinary Differential Equations, Springer, New York, NY, USA, 1998.

[15] H. L. Smith and P. Waltman, The Theory of the Chemostat, Cambridge University Press, Cambridge, UK, 1995.

[16] Z. Zhang, T. Ding, W. Huang, and Z. Dong, Qualitative Theory of Differential Equations, vol. 101 of Translations of Mathematical
Monographs, American Mathematical Society, Providence, RI, USA, 1992.

[17] L. Perko, Differential Equations and Dynamical Systems, Springer, New York, NY, USA, 2nd edition, 1996.

[18] J. K. Hale, Theory of Functional Differential Equations, Applied Mathematical Sciences, Springer, New York, NY, USA, 2nd edition, 1977.

[19] S. Ruan and W. Wang, "Dynamical behavior of an epidemic model with a nonlinear incidence rate," Journal of Differential Equations, vol. 188, no. 1, pp. 135-163, 2003.

[20] E. Beretta and Y. Takeuchi, "Global stability of an SIR epidemic model with time delays," Journal of Mathematical Biology, vol. 33, no. 3, pp. 250-260, 1995. 


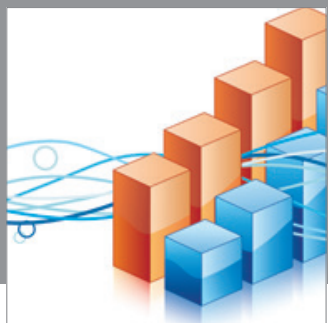

Advances in

Operations Research

mansans

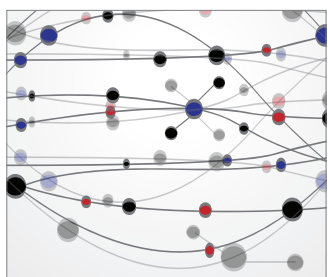

The Scientific World Journal
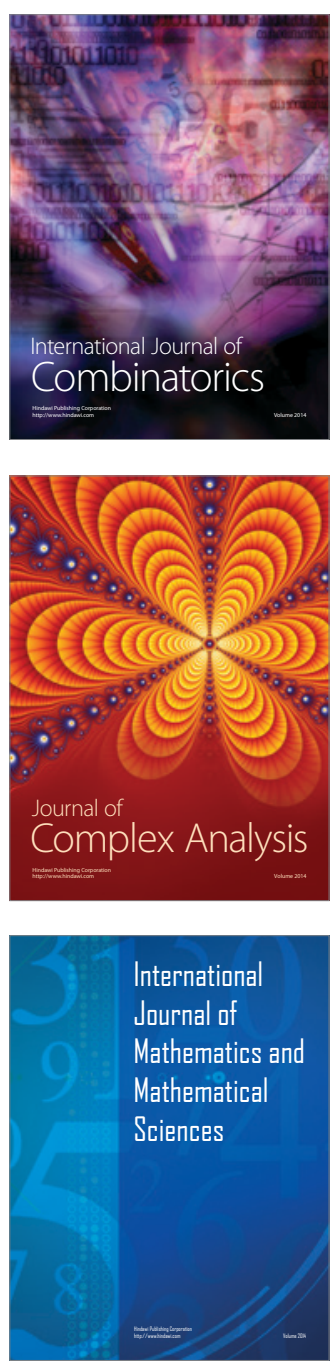
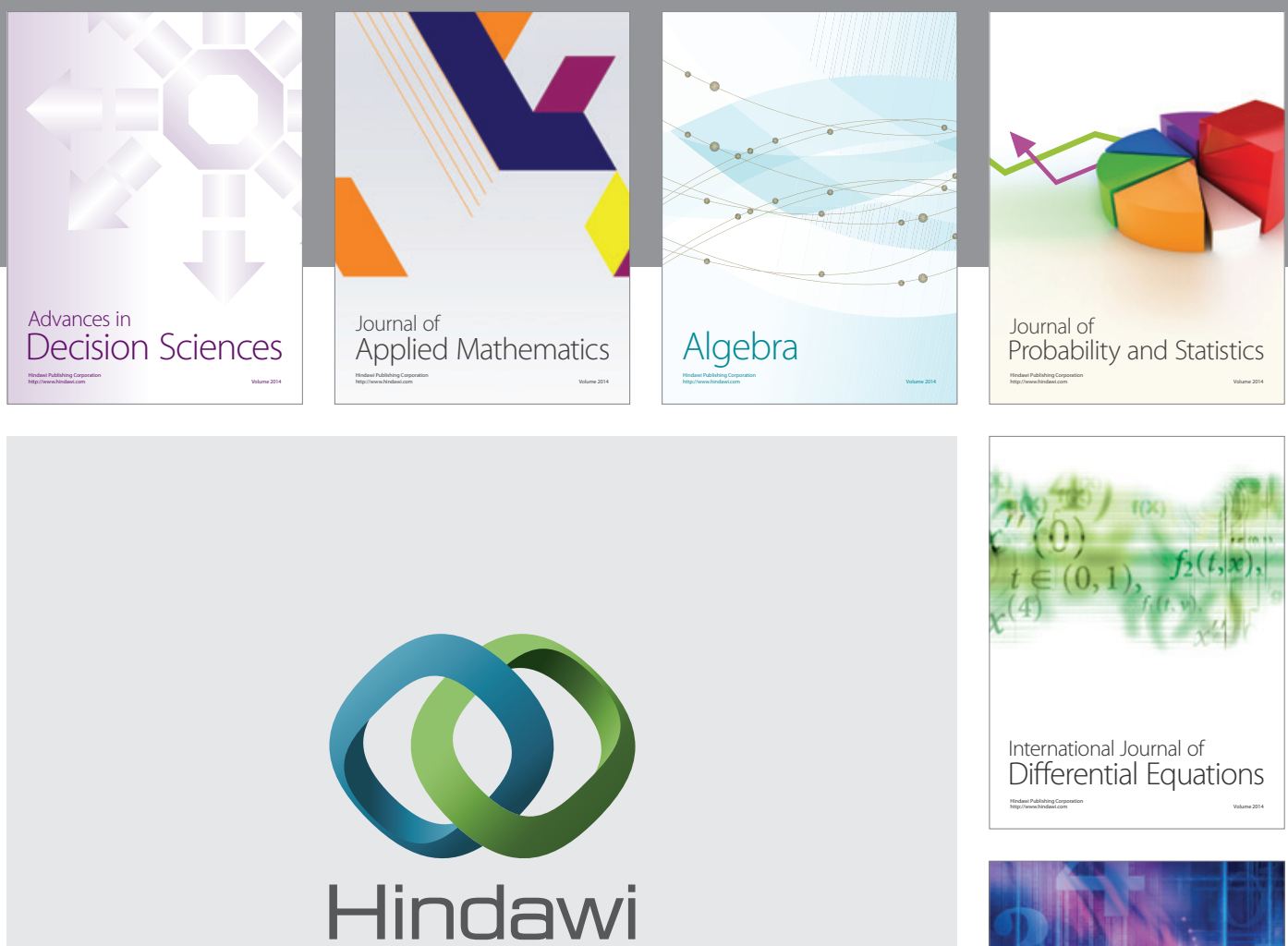

Submit your manuscripts at http://www.hindawi.com
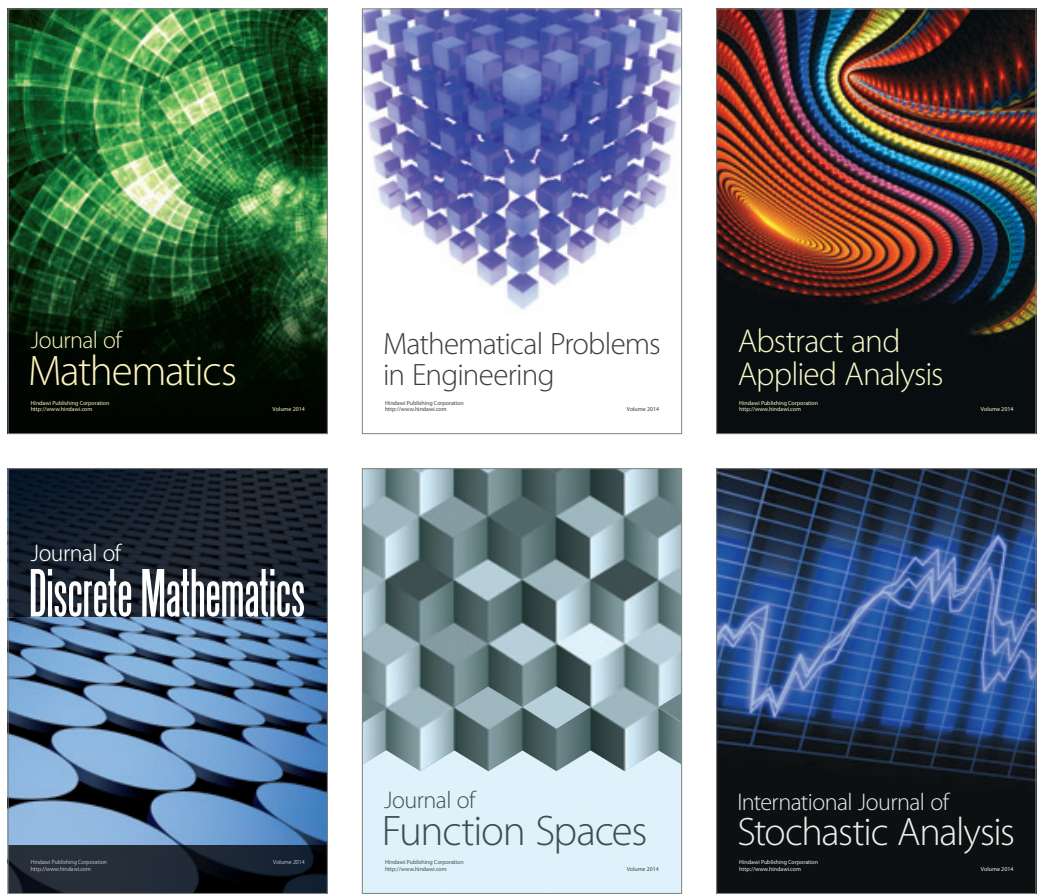

Journal of

Function Spaces

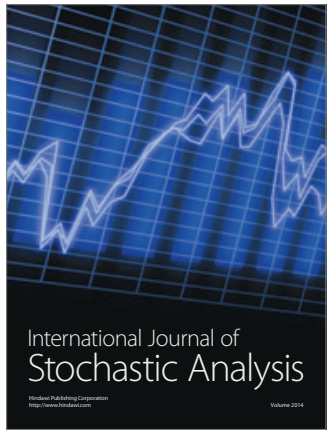

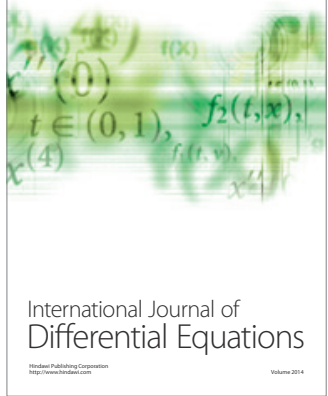
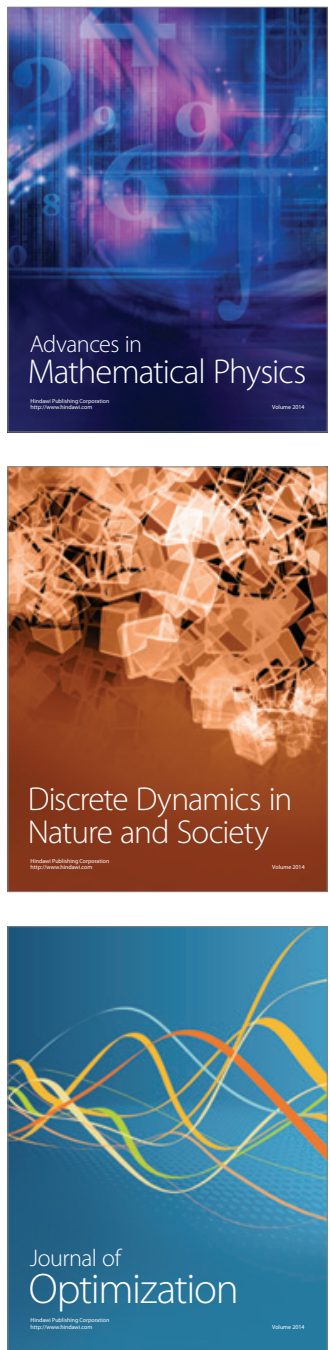